\title{
Eventos adversos: análise da equipe multiprofissional na segurança do paciente em Unidade de Terapia Intensiva
}

\author{
Adverse events: analysis of the multidisciplinary team on patient safety in the Intensive \\ Care Unit
}

Eventos adversos: análisis del equipo multidisciplinario sobre seguridad del paciente en la Unidad de Cuidados Intensivos

Emilly Jhully Correia de Paula ${ }^{1 *}$, Raquel Silva Pereira ${ }^{1}$, Debora Batista Silveira ${ }^{1}$, Leonardo Santos Silva ${ }^{1}$, Gabrielle Alves da Anunciação Barreto ${ }^{2}$, Adrielle Carvalho da Silva ${ }^{1}$, Quéren Gabriele Cunha Silva ${ }^{1}$, Gabriel Lucas Pereira Guimarães ${ }^{1}$, Nêda Lyan Souto Lopes de Queiroz¹.

\section{RESUMO}

Objetivo: Identificar e descrever os principais tipos de eventos adversos (EA) que ocorrem dentro do ambiente de Unidades de Terapia Intensiva (UTI's) juntamente com seus agentes desencadeadores e descrever a correlação entre o modelo de gestão e a postura da equipe frente aos eventos adversos e a importância de suas notificações. Métodos: Trata-se de uma revisão integrativa com abordagem descritiva qualitativa onde realizou-se um levantamento bibliográfico utilizando as bases de dados LILACS, BDENF e SciELO, utilizando os Descritores em Ciências da Saúde (DeCS). Foram encontrados 144 artigos, constituindo uma amostra final de 7 artigos. Resultados: A existência de EA na assistência prestada sinaliza o nível de qualidade do trabalho prestado nas UTI's. $O$ ato de punir diante de tal ocorrência consiste em um método que se demonstra ineficaz. Em contrapartida, a educação continuada e programas educativos sobre a segurança do paciente se mostram eficazes na redução desses incidentes, tornando a gestão hospitalar responsável por auxiliar na diminuição de fatores contribuintes à alta ocorrência de EA. Considerações finais: A gestão e a postura do gestor frente ao EA possuem influência direta na decisão dos profissionais de notificar ou não.

Palavras-chave: Unidade de Terapia Intensiva, Eventos adversos, Segurança do paciente, Notificação.

\begin{abstract}
Objective: To identify and describe the main types of adverse events $(A E)$ that occur within the Intensive Care Units (ICUs) environment together with their triggering agents and describe the correlation between the management model and the team's attitude towards adverse events and the importance of their notifications. Methods: An integrative review with a descriptive qualitative approach was carried out using LICACS, BDENF, and SciELO databases, using Health Sciences Descriptors (DeCS). 144 articles were found, constituting a final sample of 7 articles. Results: The presence of $A E$ in the care provided signals the quality level of the work implemented in the ICUs. The act of punishing such an occurrence consists of a method that is ineffective, in contrast to educational programs and continuing education on patient safety, which are effective in reducing such an occurrence, making hospital management responsible for helping to reduce contributing factors for a high occurrence of AE. Final considerations: The manager's administration and attitude towards the AE have a direct influence on the professionals' decision to notify or not.
\end{abstract}

Keywords: Intensive Care Units, Adverse events, Patient safety, Notification.

${ }^{1}$ Faculdade Adventista da Bahia (FADBA), Cachoeira - BA. *E-mail: emilly.jhullyejc@gmail.com

2 Faculdade Nobre de Feira de Santana (FAN), Feira de Santana - BA.

SUBMETIDO EM: 1/2021

ACEITO EM: 2/2021

PUBLICADO EM: $3 / 2021$ 


\section{RESUMEN}

Objetivo: Identificar y describir los principales tipos de eventos adversos que ocurren en el entorno de las Unidades de Cuidados Intensivos ( $\mathrm{UCl}$ ) junto con sus agentes desencadenantes y describir la correlación entre el modelo de gestión y la actitud del equipo ante los eventos adversos y la importancia de sus notificaciones. Métodos: Se trata de una revisión integradora con un enfoque descriptivo cualitativo donde se realizó un levantamiento bibliográfico utilizando las bases de datos LILACS, BDENF y SciELO, usando los Descriptores de Ciencias de la Salud (DeCS). Se encontraron 144 artículos, constituyendo una muestra final de 7 artículos. Resultados: La existencia de EA en la asistencia brindada indica el nivel de calidad del trabajo brindado en las $\mathrm{UCl}$. El acto de castigar ante tal hecho es un método que resulta ineficaz. En contrapartida, la educación continua y los programas educativos sobre seguridad del paciente son efectivos para reducir estos incidentes, haciendo que la dirección del hospital sea responsable de ayudar a reducir los factores que contribuyen a la alta ocurrencia de EA. Consideraciones finales: La gestión y la actitud del directivo hacia el EA influyen directamente en la decisión de los profesionales de notificar o no.

Palabras clave: Unidades de Cuidados Intensivos, Eventos adversas, Seguridad del paciente, Notificación.

\section{INTRODUÇÃO}

No início deste século o Instituto de Medicina (IOM) dos Estados Unidos da América (EUA) concerniu a segurança do paciente como um atributo significativamente relevante para a mensuração da efetividade e qualidade da assistência prestada ao paciente. Em 2004, demonstrando preocupação com a situação, a Organização Mundial da Saúde (OMS) criou a World Alliance for Patient Safety, sendo que este programa, entre vários outros objetivos, prezava não só por organizar os conceitos e definições de segurança do paciente, como propor medidas que fossem capazes de reduzir os riscos e mitigar os Eventos Adversos (EA), afinal, a assistência prestada é expressivamente humana e o ser humano está propenso ao erro, cabendo às instituições de saúde a criação de formas que diminuam as chances de que o erro atinja o paciente, se tornando um evento adverso (BRASIL, 2014).

A Portaria MS/GM n 529/2013 define evento adverso como um "incidente que resulta em dano ao paciente", sendo que incidente é definido como um "evento ou circunstância que poderia ter resultado, ou resultou, em dano desnecessário ao paciente" e a segurança do paciente como "redução, a um mínimo aceitável, do risco de dano desnecessário associado ao cuidado de saúde" (BRASIL, 2013).

Todos os setores de assistência à saúde estão sujeitos aos eventos adversos, contudo os paciente acometidos em unidades de terapia Intensiva (UTI) são particularmente vulneráveis a tais complicações e isso se dá pela oscilação no quadro clínico, que exige uma atenção maior dos profissionais envolvidos, pelo número elevado de procedimentos diagnósticos realizados, o uso de medicamentos mais complexos, tomada de decisão de alto risco, em situações de emergência e pelo alto índice de intervenções realizadas. Apesar disso ainda há uma escassez de publicações referente a segurança dos pacientes submetidos aos cuidados intensivos (NOVARETTI MCZ, et al., 2014; PEDREIRA LA, et al., 2014).

Em razão da maior suscetibilidade, tais eventos podem apresentar-se potencialmente lesivos ao indivíduo, ou até mesmo determinantes de óbito, quando em ambiente de UTI, sendo ainda associados a fatores econômicos, sociais e psicológicos, visto que também são âmbitos atingidos em decorrência dos EA e incidentes que podem acometer o cliente, não devendo ser, ser ignorados. Além disso, o aumento do tempo de internação é outra condição que traz impactos negativos, trazendo desvantagens não só ao paciente, mas envolvendo todo sistema assistencial (PEDROSA T, 2014; SOUSA A, 2018)

Pacientes hemodinamicamente instáveis apresentam uma necessidade de assistência mais rigorosa, o que inclui o uso de inúmeros materiais eletrônicos para sua prestação. Sabe-se que quanto mais complexos são os procedimentos que o paciente será submetido e a manipulação dos materiais que o mesmo necessita, maiores são as chances de que ocorra um evento adverso. As Unidades de Terapia Intensiva (UTI) constituem 
hoje um dos setores com maior prevalência de pacientes expressivamente instáveis, sendo também uma unidade que possui assistência desenvolvida em sua maior parte por procedimentos e materiais complexos, se tornando assim extremamente propenso a um alto índice de eventos adversos (ROQUE KE, et al., 2016).

Os incidentes são, em sua forma majoritária, ocasionados de forma não proposital e em incontáveis vezes o erro não é percebido pelo prestador da assistência, o que impossibilita a notificação e consequentemente diminui as possibilidades de estudo do mesmo e correção adequada através de educação continuada, por exemplo. Uma outra variável que pode ser citada como forte influenciadora nas subnotificações diz respeito ao tipo de gestão que supervisiona a assistência, já que o posicionamento dos profissionais frente aos eventos adversos sofre grande influência da mesma, sendo que esta pode ser punitiva ou não. Para além da influência exercida pela gestão vigente encontra-se também questões éticas e morais pessoais do profissional envolvido, que influenciará fortemente seu posicionamento no que diz respeito a notificação do EA ocorrido (DUARTE SCM, et al., 2014).

A ocorrência de EA cresce fortemente quando não existe por parte da gestão conhecimento das falhas de comunicação entre seus profissionais, bem como conflitos entre a equipe, afinal, isso impossibilita 0 profissional de buscar ajuda entre os companheiros de serviço para a prestação de atividades que exigem mais pessoas, bem como se sentir desconfortável em pedir ajuda na ausência de conhecimento sobre como realizar determinado procedimento, realizando assim imperícia, e consequentemente, em muitos casos, a ocorrência de um EA decorrente de tal ação (DUARTE SCM, et al., 2014).

Embora sejam considerados e denominados como fator humano, são sempre relacionados aos profissionais atuantes e quase nunca aos responsáveis pela organização de determinada instituição, mesmo após inúmeras pesquisas apontarem que os maiores propulsores de erros são fatores organizacionais, que podem ser desde estrutura organizacional até restrições financeiras. A fragmentação da responsabilidade pela assistência, problema decorrido de erros organizacionais, facilita a descontinuidade do cuidado e torna maior a ocorrência de EA, além de ocasionar falhas nas notificações e anotações, sendo estes alguns dos principais fatores associados à sua prevalência (PENA M, 2018).

Dentro do ambiente da UTI, o principal objetivo da assistência consiste em melhorar os resultados do quadro clínico do paciente, assim como também reverter seu estado de gravidade, evitando a ocorrência de complicações devido aos procedimentos do cuidado. Para que esses objetivos sejam atingidos, é necessário que ocorra uma constante avaliação da assistência prestada a estes pacientes, sendo importante que haja aceitação da existência de tais eventos e preenchimento de suas respectivas notificações, sendo estas de grande relevância para que esses erros não se tornem prevalentes (BECCARIA LM, et al., 2009).

Compreendendo a importância substancial que a notificação desempenha frente a constatação de eventos adversos e sua resolução, bem como no levantamento de necessidades institucionais de educação continuada, resolução de casos diante da justiça e possibilidades de desenvolvimento de estudos científicos, torna-se justificável a importância deste estudo. Portanto, a presente revisão objetiva identificar os principais tipos de eventos adversos que ocorrem dentro do ambiente de UTI, juntamente com os seus agentes desencadeadores, e descrever a correlação entre o modelo de gestão e a postura da equipe frente aos eventos adversos e a importância de suas notificações.

\section{MÉTODOS}

Trata-se de uma revisão integrativa com abordagem descritiva qualitativa, realizada no período de setembro a novembro de 2020, onde foram utilizadas as seguintes etapas: elaboração dos critérios de inclusão e exclusão dos artigos, seleção dos tipos de estudo para a composição do mesmo, levantamento de dados/amostra nas plataformas digitais, análise criteriosa dos dados selecionados, análise dos estudos que se encaixavam dentro dos critérios, interpretação dos resultados e exposição da revisão.

O método de Revisão integrativa tem por finalidade sintetizar resultados de um tema específico de maneira ordenada e criteriosa/sistematizada, fornecendo informações sobre um assunto ou problema à luz da literatura científica (ERCOLE FF, et al., 2014). 
No levantamento bibliográfico dos artigos, foi realizada uma busca nas seguintes bases de dados: Literatura Latino-Americana e do Caribe em Ciências da Saúde (LILACS), Base de dados de Enfermagem (BDENF), e na biblioteca virtual Scientific Electronic Library Online (SciELO). Para a busca dos artigos foram utilizados os Descritores em Ciências da Saúde: "Unidade de Terapia Intensiva", "Eventos adversos", "Segurança do paciente" e "Notificação".

Foram determinados os seguintes critérios de inclusão: artigos redigidos nos idiomas em inglês e português, artigos na íntegra que inteirem sobre a temática abordada e os que foram publicados nos últimos 11 anos. Foram excluídos dissertações, teses, capítulos de livros, editoriais, notícias e reportagens. A abordagem descritiva possibilitou observar, descrever e classificar dados reunindo conhecimentos e formulando-os de acordo com o tema abordado (Figura 1).

Figura 1 - Fluxograma das informações da pesquisa nas bases LILACS, BDENF, SciELO. Cachoeira-BA.

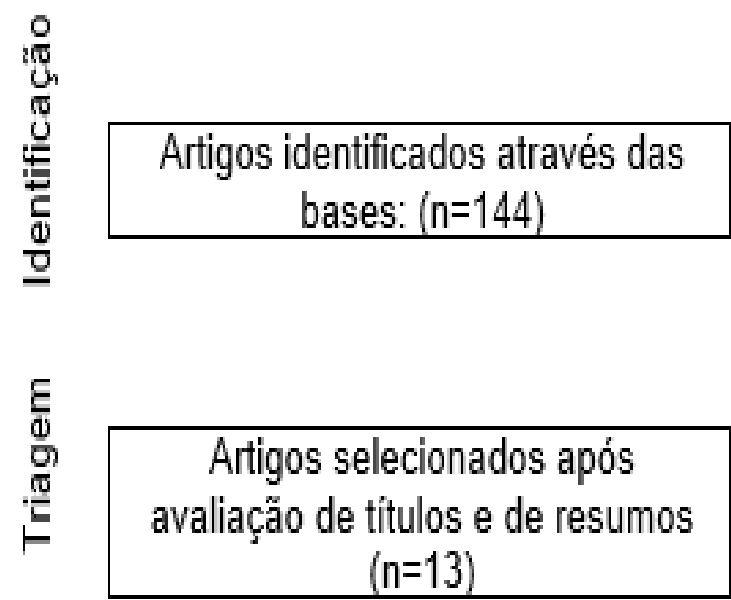

\begin{tabular}{c|}
\hline $\begin{array}{c}\text { Artigos excluídos } \\
(\mathrm{n}=119)\end{array}$ \\
\hline
\end{tabular}

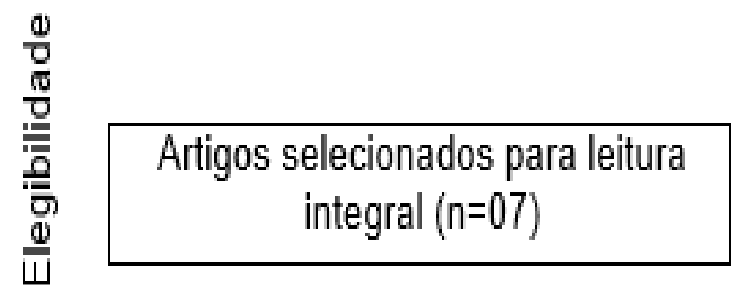

\begin{tabular}{c}
\hline $\begin{array}{c}\text { Artigos excluídos } \\
(\mathrm{n}=06)\end{array}$ \\
\hline
\end{tabular}

을

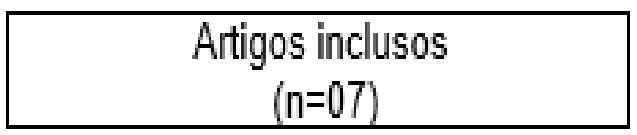

Fonte: Paula EJC, et al., 2021.

\section{RESULTADOS}

A amostra final desta revisão foi constituída por 7 artigos científicos, selecionados pelos critérios de inclusão previamente estabelecidos. O Quadro 1 apresenta as especificações de cada um dos artigos, sendo elas seus respectivos autores, título da publicação, considerações finais e revista onde os mesmos estão publicados. 
Quadro 1 - Delineamento dos estudos.

\begin{tabular}{|c|c|c|c|}
\hline Autores/Ano & Título & Considerações finais & Revista \\
\hline $\begin{array}{l}\text { CLARO CM, } \\
\text { et al., } \\
2011\end{array}$ & $\begin{array}{l}\text { Eventos adversos em Unidade de } \\
\text { Terapia Intensiva: percepção dos } \\
\text { enfermeiros sobre a cultura não punitiva. }\end{array}$ & $\begin{array}{l}\text { Embora indesejável, a cultura punitiva diante da ocorrência de EA é uma } \\
\text { realidade na UTI, indicando a necessidade de programas educativos } \\
\text { sobre a segurança do paciente voltados aos profissionais intensivistas e } \\
\text { instituições hospitalares em geral. }\end{array}$ & $\begin{array}{l}\text { Revista da Escola de } \\
\text { Enfermagem da USP }\end{array}$ \\
\hline $\begin{array}{l}\text { GONÇALVES } \\
\text { AL, et al., } \\
2012\end{array}$ &  & $\begin{array}{l}\text { Quanto maior a diferença entre as horas disponíveis e requeridas de } \\
\text { cuidado nas alocações de enfermagem, menor a frequência de EA. }\end{array}$ & $\begin{array}{l}\text { Revista da escola de } \\
\text { enfermagem da USP }\end{array}$ \\
\hline $\begin{array}{l}\text { LIMA CSP; } \\
\text { BARBOSALL } \\
\text { SFF. } \\
2015\end{array}$ & $\begin{array}{l}\text { Ocorrência de eventos adversos como } \\
\text { indicadores de qualidade assistencial } \\
\text { em unidade de terapia intensiva }\end{array}$ & $\begin{array}{l}\text { Evidenciou-se a importância no monitoramento dos eventos por meio da } \\
\text { aplicação de indicadores de qualidade com o intuito de melhoria da } \\
\text { segurança e assistência ao paciente. }\end{array}$ & $\begin{array}{l}\text { Revista Enfermagem } \\
\text { UERJ }\end{array}$ \\
\hline $\begin{array}{l}\text { ORTEGA DB, et } \\
\text { al., } \\
2017\end{array}$ & $\begin{array}{l}\text { Análise de eventos adversos em } \\
\text { pacientes internados em unidade de } \\
\text { terapia intensiva }\end{array}$ & $\begin{array}{l}\text { Houve maior incidência de eventos adversos em pacientes que exibiram } \\
\text { um perfil de maior risco e gravidade identificados por meio de escalas } \\
\text { preditoras. }\end{array}$ & $\begin{array}{l}\text { Acta Paulista de } \\
\text { Enfermagem }\end{array}$ \\
\hline $\begin{array}{l}\text { MOREIRA IA, et } \\
\text { al., } \\
2015\end{array}$ & $\begin{array}{l}\text { Conhecimento dos profissionais de } \\
\text { saúde sobre eventos adversos em } \\
\text { unidade de terapia intensiva }\end{array}$ & $\begin{array}{l}\text { Os profissionais de saúde devem ser estimulados a notificá-los e as } \\
\text { ações educativas devem ser simuladas a partir do contexto da prática em } \\
\text { saúde, como um caminho para a assistência segura e de qualidade. }\end{array}$ & $\begin{array}{l}\text { Revista Enfermagem } \\
\text { UERJ }\end{array}$ \\
\hline $\begin{array}{l}\text { BECCARIA LM, } \\
\text { et al., } \\
2009\end{array}$ & $\begin{array}{l}\text { Eventos adversos na assistência de } \\
\text { enfermagem em uma unidade de terapia } \\
\text { intensiva }\end{array}$ & $\begin{array}{l}\text { A existência de eventos adversos no cuidado prestado pela enfermagem } \\
\text { são indicadores importantes que evidenciam a qualidade da assistência } \\
\text { na unidade de terapia intensiva. Portanto, os eventos adversos devem } \\
\text { ser utilizados para subsidiar a educação permanente da equipe de } \\
\text { enfermagem. }\end{array}$ & $\begin{array}{l}\text { Rev. bras. ter. } \\
\text { intensiva }\end{array}$ \\
\hline $\begin{array}{l}\text { NOVARETT } \\
\text { MCZ, et al., } \\
\quad 2014\end{array}$ & $\begin{array}{l}\text { Sobrecarga de trabalho da Enfermagem } \\
\text { e incidentes e eventos adversos em } \\
\text { pacientes internados em UTI. }\end{array}$ & $\begin{array}{l}\text { É fundamental que os gerentes de enfermagem atuem no processo de } \\
\text { gestão de pessoas no âmbito hospitalar, evitando a sobrecarga de } \\
\text { trabalho e proporcionando, consequentemente, aumento da segurança } \\
\text { do paciente. }\end{array}$ & $\begin{array}{l}\text { Revista Brasileira de } \\
\text { Enfermagem }\end{array}$ \\
\hline
\end{tabular}

Fonte: Paula EJC, et al., 2021. 


\section{DISCUSSÃO}

\section{Principais eventos adversos e suas implicações}

Os principais EA que ocorrem dentro do setor da UTI estão relacionados à assistência prática prestada com frequência ao paciente. Observa-se que eventos como úlcera por pressão/lesão por pressão (LPP), extubação acidental, falha de registro em prontuário, especialmente as anotações de enfermagem, seguida de erro no preparo e administração de medicamentos, perda de sondas gastroenteral, sonda nasoenteral e a perda de cateter venoso central são alguns dos eventos mais notificados neste ambiente. Os eventos supracitados impactam diretamente na ocorrência de uma margem de erro significativa na avaliação dos prontuários, dificultando a assistência da enfermagem no serviço de auditoria interna e externa (GONÇALVES LA, et al., 2012; LIMA CSP, 2015; ORTEGA DB, et al., 2017; MOREIRA IA, et al., 2015).

Estando também entre os EA em UTI's mais comuns, as caligrafias ilegíveis dos médicos, a distração dos profissionais durante manuseio de equipamentos e realização de tarefas. O procedimento de cabeceira elevada é fundamental em ambiente de terapia intensiva em pacientes intubados, essa assistência simples tem sido de difícil realização pelos profissionais do setor, além da ausência de verificação de pressão venosa central (CONTRIN M, et al., 2009).

As reações adversas, lesões temporárias e permanentes e até mesmo a morte do paciente em casos mais graves, são apontados como prejuízos/danos que advêm da ocorrência de erros na administração de medicamentos. A falta de registro correto é um ponto que corrobora diretamente para a eventualidade desses erros. Como exemplo temos os erros na prescrição médica e também na anotação do balanço hídrico que inviabilizam a continuidade do cuidado ou/e atrasam o processo assistencial, além de gerarem maior propensão ao erro, além de procedimentos de curativos não realizados e paramentação correta (MOREIRA IA, et al., 2015; CONTRIN M, et al., 2009).

São os profissionais de enfermagem e fisioterapia que realizam a maioria dos procedimentos que acabam por resultar em eventos adversos, assim como também são eles que estão em constante contato com os pacientes dentro das UTIs, por isso percebe-se a importância da Sistematização das ações da enfermagem e de toda a equipe multiprofissional na assistência efetiva e na prevenção de EA durante 0 manejo de pacientes graves, assim como também guias ou protocolos como o Protocolo Operacional Padrão (POP), para que, além da assistência qualificada, possam ser identificados fatores de riscos e minimizar a incidência dos EA para melhorar o cuidado e garantir a segurança do paciente (LIMA CSP, 2015; KIEKKAS P, et al., 2013; RAMALHO JMN, et al., 2014).

\section{Caracterizando os principais fatores de riscos para eventos adversos}

A UTI, por ser um ambiente de experiências angustiantes e de tensão constante, mostra-se prejudicial à organização do trabalho e à saúde da equipe assistencial (especialmente da enfermagem). Dessa forma, os artigos analisados trazem que há uma maior taxa de eventos que ocorrem em ambiente de terapia intensiva do que nos demais. $E$ apresentam como sendo principais fatores causadores de $E A$, além da ilegibilidade da caligrafia médica, as intercorrências que surgem em horário de trabalho e que levam à distração e à sobrecarga do profissional (MOREIRA IA, et al., 2015; NOVARETTI MCZ, et al., 2014).

Segundo Moreira IA, et al., (2015) para além da presença do ser humano nos EA, outras condições podem estar relacionadas à ocorrência de erros, tais como: sobrecarga de trabalho, atividades realizadas na unidade e fatores estruturais da unidade. Agrega-se ainda a esse cenário o constante avanço tecnológico e a ausência de educação continuada adequada às necessidades ou incompatibilidade profissional a esse avanço, tornando profissionais antes capazes a desatualizados pelos novos métodos e forma de cuidar, entre outras situações.

Tais fatores estão estritamente relacionados à alocação de enfermagem, sendo possível observar que quando o tempo de disponibilidade da enfermagem é inferior ao tempo de cuidado que o paciente requer, há maior frequência de EA (MOREIRA IA, et al., 2015; GONÇALVES LA, et al., 2012). Ademais, também relacionada à sobrecarga profissional, uma realidade vivida pelos profissionais de enfermagem é a dupla 
jornada de trabalho frente a uma baixa remuneração, que desencadeia um nível elevado de estresse nos profissionais prejudicando não só a assistência prestada, mas também trazendo males a saúde do trabalhador (NOVARETTI MCZ, et al., 2014).

O quantitativo de enfermeiros trabalhando em UTI's colabora na distribuição de atividades a serem realizadas, quando o número de profissionais é suficiente diminui os riscos de possíveis efeitos adversos devido a execução de tarefas com tempo necessário para ser prestado com qualidade, evitando correrias e imprevistos (ORTEGA DB, et al., 2017). Levando em consideração outros fatores de risco, é de grande valia destacar o uso incorreto ou a falta de EPIs pelos profissionais, quando se deveria estar devidamente paramentado, além dos eventos adversos decorridos do manuseio incorreto de equipamentos. Outro fator, associado aos previamente apresentados, são as falhas e inconsistências nas anotações da equipe de enfermagem, além de erros de cálculos de balanços hídricos e preenchimentos parciais dos mesmos ou dados ignorados (MOREIRA IA, et al., 2015).

A idade avançada, longo período de internação, escores de maior gravidade clínica e maior risco para lesão por pressão foram apontados como alguns dos principais agentes causais para a incidência dos EA (ORTEGA DB, et al., 2017). Há uma incidência de quase 11 vezes mais na probabilidade da ocorrência dos EA nas admissões que se prolongam por um período maior que quatro dias em relação às admissões mais curtas. Além disso, nas admissões em que os profissionais de enfermagem sobrecarregados acompanharam, a chance da ocorrência dos EA foi o dobro (BECCARIA LM, et al., 2017; NOVARETTI MCZ, et al., 2014).

A sobrecarga dos profissionais, apontada como um importante fator de risco para a ocorrência de EA, sofre grande influência, positiva ou negativa, da gestão do serviço e sua capacidade de realizar um bom planejamento, controle e supervisão do trabalho relacionado às demandas na previsão, provisão e também na manutenção dos equipamentos e recursos materiais e humanos (BECCARIA LM, et al., 2017; NOVARETTI MCZ, et al., 2014).

\section{Postura profissional frente aos eventos adversos e sua relação com a subnotificação}

O erro no campo da saúde é visto como um ato vergonhoso que acarreta aos profissionais olhares de incompetência e desatenção (MOREIRA IA, et al., 2015). BECCARIA LM, et al. (2009), em seu estudo relacionado a EA na assistência de enfermagem em UTI, estimou que apenas $37,83 \%$ dos profissionais alegam relatar o ocorrido ao enfermeiro ou supervisor da unidade. $O$ baixo entendimento dos profissionais de saúde sobre o EA e a importância de ser relatado demonstram a falha das políticas gerenciais na busca por métodos que elevem o conhecimento da equipe multiprofissional e os orientes a notificar ao Serviço de Gerenciamento de Riscos.

Em seu estudo, Claro CM, et al., (2011) estima que 71,4\% profissionais de enfermagem participantes assumem a existência de subnotificação de EA e pontuam que tal fato está diretamente relacionado ao descrédito desses eventos, sobrecarga de trabalho, esquecimento, além de sentirem vergonha de realizar a notificação; o autor relata que $70 \%$ dos profissionais participantes do estudo afirmaram ter conhecimento da existência do sistema de notificação de EA, salienta-se que $83,7 \%$ dos profissionais responsáveis por realizar a notificação de EA eram enfermeiros.

Durante um EA a equipe preocupa-se mais em apontar o culpado do que iniciar medidas de prevenção para novas ocorrências, desestimulando a notificação sistemática e o uso dos instrumentos padronizados que realizam análise dos eventos ocorridos. Sendo tomadas poucas medidas por parte do setor administrativo frente ao profissional que comete o EA, não sendo realizadas condutas como a advertência verbal, esclarecimento do erro e anotações no prontuário do paciente (BECCARIA LM, et al., 2009).

A notificação consiste em parte extremamente importante para que os EA não se tornem predominantes, pois através da mesma é possível se avaliar os tipos de erros mais prevalentes e trabalhar medidas para alterar a situação. Entretanto, a cultura punitiva frente ao erro ainda existe, e consiste em grande fator agravante para que haja subnotificação do EA. O posicionamento do profissional frente ao EA sofre alterações significativas baseado no tipo de gestão vigente na instituição, podendo constatar que enquanto em algumas 
instituições os enfermeiros que referem ter vivenciado a experiência e não ter sofrido represália possuem segurança para notificar a ocorrência de EA, em outras instituições os profissionais referem ter recebido punição e não possuir segurança para realizar a notificação, sendo predominante a advertência verbal feita no próprio local de trabalho (CLARO CM, et al., 2011).

\section{Medidas para diminuição dos eventos adversos}

A maneira mais prática para se reduzir a prevalência dos erros se baseia na realização constante e periódica de educação permanente, principalmente se tratando de erros relacionados à administração de medicamentos. Para além disso, simplificar e padronizar os processos, desde a realização de prescrição médica eletrônica e sem abreviações, bem como da distribuição da farmácia em horários padrões e mediante supervisão farmacêutica, e por fim, padronização da administração de medicamentos por meio de criação de protocolos, consistem em medidas frequentemente apontadas como capazes de diminuir significativamente a recorrência dos erros. É imprescindível que as instituições de saúde elaborem protocolos assistenciais ao paciente e capacitem toda a sua equipe de saúde, através de medidas de educação continuada com o intuito de melhorar os indicadores de saúde (BECCARIA LM, et al., 2009; MOREIRA IA, et al., 2015).

Percebe-se que a prática de ações educativas direcionada aos profissionais que atuam diretamente na assistência favorece o aumento da qualidade do cuidado prestado ao cliente. Alguns eventos adversos como as LPP destacam-se por serem considerados como preveníveis pela adoção de medidas preventivas protocoladas pela gestão como monitoramento, inspeção e proteção da pele, além de mudança de decúbito, evitando a recorrência de erros e desenvolvendo, assim, uma cultura de segurança. Um exemplo de protocolo a ser usado é a escala de BRADEN, que avalia a presença de riscos para desenvolvimento dessas lesões, prevenindo que as mesmas aconteçam (BECCARIA LM, et al., 2009; LIMA CSP, 2015).

Pesquisa realizada em enfermarias cirúrgicas de um hospital universitário do Japão apresentou resultados semelhantes aos do presente estudo, demonstrando que encontros educativos breves, com duração de 15 minutos, durante 6 meses sobre a segurança do paciente, aumentou a taxa de notificação; no entanto, o efeito da intervenção diminuiu após 6 meses da realização da educação, reforçando a necessidade de educação continuada a longo prazo, para manter os resultados positivos tendo como objetivo a melhoria contínua e a avaliação da qualidade da assistência e os possíveis problemas relacionados às falhas do sistema como falta de liderança, objetivos não traçados ou alcançados e até mesmo lacunas nas estratégias do cuidado (BECKMANN U, et al., 1996).

É necessário que ocorra uma monitorização constante das atividades desde seu planejamento até a realização das mesmas para que assim sejam identificados possíveis problemas ou riscos potencialmente desencadeadores de EA, as causas subjacentes e os fatores contribuintes sendo, no entanto, erradicados (BECKMANN U, et al., 1996).

\section{CONSIDERAÇÕES FINAIS}

O ambiente hostil encontrado nas unidades de terapia intensiva tende a favorecer a ocorrência de EA, especialmente quando associados a internações de longa duração e/ou pacientes idosos. A subnotificação impossibilita que o gestor tenha um panorama fidedigno da prevalência dos EA dentro da instituição e consequentemente inviabiliza que aconteça educação continuada diretamente proporcional à ocorrência de EA, tornando assim os erros mais prevalentes. O modelo da gestão e a postura do gestor frente ao EA e sua notificação possuem influência direta na decisão de relatar ou não um evento adverso da parte dos profissionais assistenciais.

\section{REFERÊNCIAS}

1. BECCARIA LM, et al. Eventos adversos na assistência de enfermagem em uma unidade de terapia intensiva. Revista Brasileira de Terapia Intensiva, 2009 21(3): 0103-507X

2. BECKMANN U, et al. The australian incident monitoring study in intensive care: AIMS-ICU. The Development and evaluation of an incident reporting system in intensive care. Anaesth Intens Care. 1996; 24(3):314-9. 
3. BRASIL. Programa nacional de segurança do paciente. 2014 . Disponível em: https://bvsms.saude.gov.br/bvs/publicacoes/documento_referencia_programa_nacional_seguranca.pdf. Acessado em: 25 de janeiro de 2021.

4. BRASIL. PORTARIA № 529, DE 1ํ DE ABRIL DE 2013. 2013. Disponível em: https://bvsms.saude.gov.br/bvs/saudelegis/gm/2013/prt0529_01_04_2013.html. Acessado em: 25 de janeiro de 2021.

5. CLARO CM, et al. Eventos adversos em Unidade de Terapia Intensiva: percepção dos enfermeiros sobre a cultura não punitiva. Rev. esc. enferm. São Paulo. 2011; 45(1):167-172.

6. CONTRIN M, et al. Eventos adversos na assistência de enfermagem em uma unidade de terapia intensiva. Revista brasileira terapia intensiva, 2009; 21(3): 276-282.

7. DUARTE SCM, et al. Eventos adversos e segurança na assistência de enfermagem. Revista Brasileira Enfermagem, 2015; 68(1):144-154.

8. ERCOLE FF, et al. Revisão integrativa versus revisão sistemática, Nursing Journal of Minas Gerais, 2014; 18(1):9-12.

9. GONÇALVES LA, et al. Alocação da equipe de enfermagem e ocorrência de eventos adversos/incidentes em unidade de terapia intensiva. Revista Brasileira Enfermagem, 2012; 46 :71-77.

10. KIEKKAS P, et al. Unplanned extubation in critically ill adults: clinical review. Nursing in Critical Care, 2013; 18: 12334.

11. LIMA CSP, BARBOSA SFF. Ocorrência de eventos adversos como indicadores de qualidade assistencial em unidade de terapia intensiva. Uerj Nursing Journal, 2015; 23(2): 222-8.

12. MOREIRA IA, et al. Conhecimento dos profissionais de saúde sobre eventos adversos em unidade de terapia intensiva. Uerj Nursing Journal, 2015; 23(4): 461-7.

13. NOVARETTI MCZ, et al. Sobrecarga de trabalho da Enfermagem e incidentes e eventos adversos em pacientes internados em UTI. Revista brasileira de enfermagem. 2014; 67(5): 692-699.

14. ORTEGA DB, et al. Análise de eventos adversos em pacientes internados em unidade de terapia intensiva. Acta Paul Enfermagem, 2017; 30(2):168-173.

15. PEDREIRA LA, et al. Evento adverso no idoso em Unidade de Terapia Intensiva. Revista brasileira Enfermagem, 2013; 429-436.

16. PEDROSA T, COUTO R, Erros e eventos adversos na assistência médico-hospitalar. Revista Médica de Minas Gerais, Minas Gerais, 2014; 24(2): 216-222.

17. PENA M, MELLEIRO M. Eventos adversos decorrentes de falhas de comunição: reflexões sobre um modelo para transição o cuidado. Revista de enfermagem UFSM, 2018; 8(3): 616-625.

18. RAMALHO JMN, et al. Extubação acidental e cuidados intensivos de enfermagem. Rev enferm UFPE on line., 2014; 8: 3945-52.

19. ROQUE KE, et al. Eventos adversos na unidade de terapia intensiva: impacto na mortalidade e no tempo de internação em um estudo prospectivo. Cad. Saúde Pública, 2016; 32(10): e00081815.

20. SOUSA, A. Eventos Adversos nas Unidades de Tratamento Intensivo. Revista Científica Multidisciplinar Núcleo do Conhecimento, 2018; 08(11): 105-116. 